\title{
Do some cases of anorexia nervosa reflect underlying autistic-like conditions?
}

\author{
C. Gillberg and M. Råstam \\ Department of Pediatrics and Child Psychiatry, Child Neuropsychiatry Centre, Annedals \\ Clinics, S-413 45 Göteborg, Sweden
}

Correspondence: C. Gillberg at above address

\begin{abstract}
In a sample of 51 teenagers with anorexia nervosa (AN)—which included 24 cases constituting the total population of AN cases born in 1970 - several had shown social, communicative and behaviour patterns suggestive of autistic-like conditions as children, long before the onset of AN. One of the three boys in the AN group had Asperger syndrome. Three of the 48 girls had histories suggesting high functioning autism and continued to show many features typical of autism. Two further girls had Tourette syndrome and obsessive-compulsive traits in combination with social interaction problems. Eighteen other girls met criteria for obsessive-compulsive personality disorder (OCPD) and most of these also had had moderate-severe childhood social interaction problems. In a sex- and age-matched comparison group from the same schools, two girls had OCPD, but none had autistic-like conditions or Tourette syndrome. The results are discussed in the context of a recently suggested link between Asperger syndrome, Tourette syndrome and obsessive-compulsive problems, and it is suggested that AN in a subgroup of cases might represent a disorder belonging in the same class as autism and autistic-like conditions.
\end{abstract}

\section{INTRODUCTION}

Anorexia nervosa is often accompanied by obsessive and compulsive symptoms, sometimes amounting to a fullblown obsessive-compulsive disorder (OCD), even if one does not count manifestations directly associated with the eating disorder as such (Dally, 1969). Furthermore, obsessive-compulsive personality disorder (OCPD) is often evident in the premorbid histories of young patients with anorexia nervosa (Råstam, 1992).

The literature abounds with references to "obsessions" and "compulsions" being common phenomena in children suffering from autism (e.g. Rutter, 1985). Indeed ritualistic and compulsive behaviours are considered by most authors to be excellent examples of the type of restricted behaviour which is included as a criterion for diagnosing autism (Rutter, 1978; APA, 1980, 1987). Baron-Cohen recently suggested that "the terms obsession and compulsion should be used with considerable caution to describe autistic behaviours", because of the impossibility-in autism - to decide whether the symptoms are "egodystonic" or not (Baron-Cohen, 1989). However, many patients with "true" obsessive-compulsive disorders demonstrate the same types of relatively meaningless restricted behaviour patterns typically encountered in autism. Not all of these behaviours can be regarded as "egodystonic". The DSM-III-R (APA, 1987) particularly notes that the recognition that compulsive behaviour is

C)1992 Rapid Communications of Oxford Ltd excessive or unreasonable ("egodystonic") may not exist in "young children".

The essential feature of "obsessive-compulsive personality disorder" (OCPD) is a "pervasive pattern of perfectionism and inflexibility" (APA, 1987). An egodystonic quality is not required for this diagnosis to be made. In fact, it is implicit that, in many cases, the perfectionistic traits are rather egosyntonic. In any case, the clinical distinction of egosyntonic/egodystonic in this field, seems to us to reflect remnants of a psychodynamic theoretical framework which may not have much to contribute to the understanding of some of the more severe forms of OCPD and OCD.

The "inflexibility" of OCPD is also typical of autism. Many autism symptoms (perhaps the majority) can be regarded as signs of an inflexibility in all aspects of relating to the environment. A comparison of the symptoms of OCPD listed in the DSM-III-R and the diagnostic criteria for autistic disorder in that manual reveals that all nine OCPD symptoms could be counted as examples of behaviours signalling "qualitative impairment in reciprocal social interaction" and "markedly restricted repertoire of activities and interests", two of three main symptom groups typifying autism.

Obsessive-compulsive symptoms occur in a majority of patients with Tourette syndrome (Comings, 1990).

Behavioural Neurology . Vol 5 . 1992 27 
Quite often, such phenomena are severe enough to warrant a diagnosis of OCD or OCPD. Tourette syndrome, on the other hand, has been described in connection with autism, pervasive developmental disorders (APA, 1987) and Asperger syndrome relatively often over the last 10 years (e.g. Littlejohns et al., 1990; Comings and Comings, 1990), and this has lead to speculation that Tourette and autistic-like conditions may have other than coincidental links.

A link between anorexia nervosa and autism was proposed some years ago (Gillberg, 1985), when three families of male probands with autism were reported to contain second degree female relatives with anorexia nervosa. In particular, the obsessive-compulsive quality of both conditions was highlighted in an attempt to find possibly unifying features.

The present study reports findings from a major study of anorexia nervosa, which included a total population of anorexia cases from one birth cohort and an individually sex-, age-, and school-matched comparison group. A high rate of pre-morbid social impairment and obsessive-compulsive personality disorder was found in the anorexia group. The details in this respect are reported below.

\section{MATERIAL AND METHODS}

\section{Anorexia nervosa group}

The Anorexia nervosa group consisted of 51 cases ( 3 males, 48 females), 10-17 years of age at onset of the eating disorder. A vast majority of these cases were psychiatrically examined within 18 months of the onset of anorexia nervosa. The mean age at the time of psychiatric examination was 16 (95\% confidence limits 15.5-16.5) years. Almost half of this group $(n=24)$ consisted of the total population of AN cases born in 1970 and living in Göteborg before their 18th birthday, less one girl who refused participation in the psychiatric examination. (Details of the population screening and psychiatric study are available in Råstam et al. (1989) and Råstam (1992).) The other half comprised a mixture of population screening and referral cases. The two groups were compared on several hundred different background measures and were found to be very similar in almost all respects. It was therefore decided to pool them for the further presentation of results. Forty-nine of the cases fulfilled DSM-III-R criteria for anorexia nervosa (APA, 1987). Thirty-four of these also met DSM-III criteria for the disorder (APA, 1980). Three cases were diagnosed as "partial anorexia nervosa" on the basis that they only just failed to fulfil DSM-III or DSM-III-R criteria. Apart from the girl in the population group who refused to participate, there was no attrition, and so 51 cases remained for psychiatric examination.

\section{Comparison group}

All 51 cases in the Comparison group were individually matched for age, sex and school with the children in the anorexia nervosa group who participated in the psychiatric study.

\section{Psychiatric examination}

Of the 102 girls and boys in the study, 100 were examined by the second author and two by the first (those two who refused to be examined by anybody but the head of the department). The examinations took place in an outpatient clinic in 98 cases, in the homes of the teenagers in three and in a child psychiatric clinic in one.

The teenagers brought their mothers in 98 cases, their fathers (who was the responsible caregiver) in two cases, and came alone in two cases. The child and parent were first seen together. After that, the mother was interviewed for several hours. At the same time, the child was measured, had blood pressure taken and was given questionnaires to complete in another room. The mother was then asked to complete questionnaires. In a separate room, the child was at the same time physically and psychiatrically examined. All the parents and children (except two, see above) were examined by the second author.

\section{Structured interview manuals}

A semi-structured interview-directed to the responsible caregiver-which has been used in a number of population-based studies in Göteborg throughout the last 15 years was adapted for use in the present study. It concerns mother's experience of pregnancy and mother-infant interaction, detailed aspects of the child's health and development, the child's temperamental style, interests and peer relationships, interaction with other family members, rearing conditions, social conditions and hereditary factors.

A Swedish version of the FACES questionnaire (Olson et al., 1979; published in Råstam and Gillberg, 1991), developed for the evaluation of adaptability and cohesion in family communication, was given both the parent and child. Results from this study have been published separately (Råstam and Gillberg, 1991).

A Swedish translation of the EAT (Eating Attitudes Test) (Garfinkel and Garner, 1979) was given for completion to the parent and the child separately.

The Eysenck Personality Inventory (Eysenck and Eysenck, 1964) was completed by the parent.

The Birleson Depression Inventory (Birleson, 1981) has been translated into Swedish. It was given to all the probands and comparison cases in the present study.

\section{Physical examination}

A neurodevelopmental assessment as outlined in Gillberg (1986) was made of all the probands and comparison 
cases. This comprised, among other things, a detailed assessment of the performance of diadochokinesis (alternating pro-supination) with recording of time, number of pro-supinations, smoothness and associated movements.

\section{Blind study of pre-morbid psychiatric problems and personality disorders}

On the basis of all the results obtained during the examinations outlined (including the psychiatric interview and self-report and parent questionnaires), case-notes were prepared for each child. These concerned the temperamental, behavioural, emotional and physical characteristics of the child up to about age 10 years. The case-notes were prepared by the second author and were de-identified and deleted of all information pertaining to the diagnosis of anorexia nervosa. They were sorted randomly, so as not to raise suspicion of overall group status (Anorexia nervosa or Comparison), and given to the first author for diagnosis of pre-morbid personality and psychiatric problems of various kinds. The DSM-III-R (APA, 1987) was used for assigning diagnoses, except in the case of Asperger syndrome and autistic-like conditions. For these diagnoses, the criteria of Gillberg and Gillberg (1989) and Steffenburg and Gillberg (1986) were used instead. For obvious reasons, the interviewer could not remain blind to whether the teenager had anorexia nervosa or not.

\section{Friendship and social interaction skills}

All parents and children were interviewed-in structured ways-about the children's ability to make friendships and whether or not they had any close friends (or just acquaintances). The second author (who performed almost all of these interviews) contended that parents were more likely to "overvalue" the children's capacities in these respects than were the children themselves, who appeared to give accurate accounts of their relationships with friends and other people. The children's information was therefore used in the presentation of results. Parents, on the other hand, tended to be able to describe their children's general ability to interact with other people ("Does your child interact socially with other people without difficulty or is there any kind of social impairment?").

\section{RESULTS}

Overall frequency of autistic-like and obsessivecompulsive disorders

According to the blind case-note study of premorbid problems, altogether 24 cases in the Anorexia nervosa group compared with two in the Comparison group fulfilled criteria for OCPD, Asperger syndrome, autistic-like conditions or Tourette syndrome $(p<0.001)$ (Fig. 1). Two further children in the Comparison group had shown some deficits in attention, motor control and perception (including some behaviours reminiscent of those seen in autisticlike conditions) in early childhood, but were free of such problems already before age 10 years. Many of the remaining anorexia cases were described as perfectionistic and "obsessive" without fulfilling criteria for OCPD or OCD. Some of these results have been published before (Råstam, 1992), but will be described in greater detail here as they relate to traits belonging in the autism spectrum.

Individual cases with autistic-like and obsessivecompulsive premorbid problems

One boy (out of three boys) in the Anorexia nervosa group had typical Asperger syndrome.

Three girls with anorexia nervosa had premorbid autistic-like conditions. All three had severe impairment of reciprocal social interaction, extreme difficulties making friends, odd prosody, monotonous voice and/or pedantic speech and obsessive-compulsive rituals of various kinds

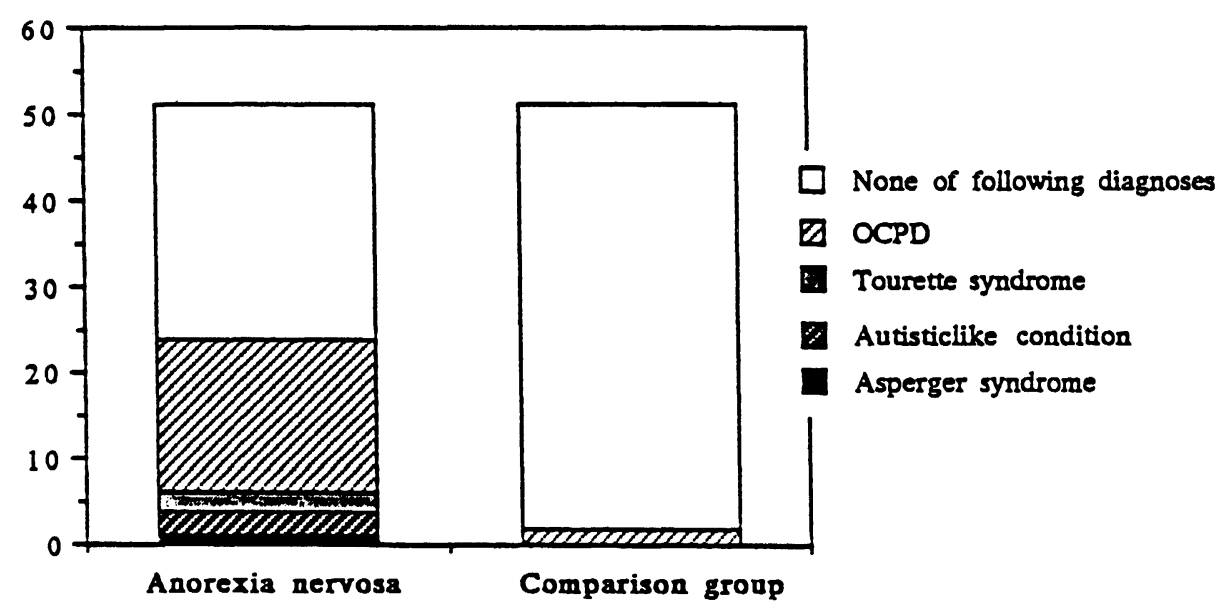

FIG.1. Autism—obsessive-compulsive spectrum problems in anorexia nervosa and comparison cases. 
at least from age 5 to age 10 years. All three still exhibited similar problems, but one fulfilled criteria for schizotypal personality disorder and another one fitted the picture of histrionic personality disorder also. It was not possible to document clearly a typically autistic development during the first 2-3 years. They were therefore classified as "autistic-like" in spite of the fact that all three seemed to be fairly characteristic of high-functioning autism, once such a diagnosis was considered.

Two girls in the Anorexia nervosa group had Tourette syndrome. Both of these had severe impairments of reciprocal social interaction from early childhood, they showed abnormalities of speech production (monotonous voice) and were characterised by obsessive-compulsive traits.

Eighteen girls in the Anorexia nervosa group and two in the Comparison group met criteria for OCPD $(p<0.01)$. Of the Anorexia girls with OCPD, 13 (72\%) had shown major peer friendship problems from early childhood. Seven of these girls also showed abnormalities of speech production (such as odd prosody, monotonous voice, irreverent or pedantic speech). Their rituals and compulsions were often very similar to those encountered in autism (attachments to odd objects or people for example).

\section{Some hereditary/familial factors in the autistic-like disorders}

The boy with Asperger syndrome had a mother who was given this diagnosis in connection with the psychiatric examination. His father, who had committed suicide, was described both by the mother and the patient as clearly Asperger-like.

One of the girls with an autistic-like condition had a mother with classical Asperger syndrome and a maternal aunt with possibly Asperger syndrome (according to the description given by her sister). A male maternal first-cousin of the proband had been a severe arsonist already at the age of 10-11 years. The father had several traits reminiscent of Asperger syndrome (according to the mother's description), but also showed a strong tendency for mood swings. The paternal grandmother had suffered from Alzheimer type dementia.

Another one of the girls with an autistic-like condition had a father who was described by his wife as "odd, aloof and indifferent". She also had a brother and sister with conduct disorder, gaze avoidance and lack of imitation skills in early childhood. The paternal grandmother had been treated in hospital because of an affective disorder. The maternal grandfather was described as possibly having Asperger syndrome. His sister, in turn, was described as "very odd".

The third girl with an autistic-like condition had a mother with relapsing depressions and phobias and a maternal grandfather with alcoholism. A maternal aunt had an anxiety state and the daughter of an elder brother of the patient's had shown severe conduct problems and attention deficits in childhood.

Since so few fathers attended, we took a cautious attitude in assigning possible psychiatric diagnoses to them. We argued that in cases with major problems (such as in the families with anorexia children) there might, theoretically, be a tendency for some of the mothers to exaggerate any problems their husbands might have. Nevertheless, the general impression was that this was not common. Several mothers described their husbands as having Asperger type problems. These were mothers of anorexic girls with OCPD and autistic-like conditions.

Two brothers of two healthy girls in the Comparison group were described by their mothers to have some Asperger traits also. However, these boys did not appear to be severely handicapped by these traits.

\section{Dysdiadochokinesis}

Dysdiadochokinesis was present in 19 of the Anorexia nervosa group cases and in six of the Comparison group cases $(p<0.02)$. This symptom was particularly common in the 24 anorexia cases with Asperger syndrome, autisticlike conditions. Tourette syndrome or OCPD $(n=11)$ and slightly less common in the 27 anorexia cases without such diagnoses $(n=8)$ (n.s.). All four cases with Asperger syndrome or autistic-like conditions had dysdiadochokinesis. A few other measures of gross and fine motor performance were used, but no differences across groups emerged. The majority of the girls and boys in the study were not emaciated at the time of examination. Thus, dysdiadochokinesis in the anorexia group could not be accounted for by overall malfunction of the brain caused by starvation.

\section{Friendships/social relationships/spontaneous imaginative activity}

There were 32 cases in the Anorexia nervosa group who admitted to having had problems in peer relationships before age 10 years (long before the onset of anorexia nervosa). They either had no peers or acquaintances at all or had only superficial acquaintances. The corresponding number in the Comparison group was $8(p<0.001)$.

In connection with the interview, the teenagers were asked to make a drawing and to write a spontaneous essay about themselves. So far this essay has not been subjected to systematic study, but the impression was that the anorexia nervosa cases had much greater difficulty than the comparison cases when it came to describing not only their own feelings, but also their own thoughts and the thoughts and feelings of others.

\section{DISCUSSION}

Teenage onset anorexia nervosa is by many considered to be a disorder signifying pubertal crisis of one or other kind 
(Crisp, 1980). The implication of our findings is that anorexia nervosa might-at least in an important minority of the cases-be one relatively late outward manifestation of a neuropsychiatric disorder which has been present from early childhood and characterised by social interaction problems, communication pecularities, obsessiveness, compulsions and restricted interests. These symptoms are generally considered the hallmarks of autism and autisticlike conditions.

There are several reasons why the findings cannot be summarily dismissed as pure chance coincidences. First of all, the Anorexia nervosa group is possibly more representative of teenage anorexia nervosa than are almost all previously published cohorts of anorexia cases (Råstam et al., 1989). Our group included the total population of anorexia cases born in 1970, and the remaining cases, even though not purely population-based, were similar in all key respects to the population group. This argues in favour of the Anorexia nervosa group being representative. Second, we have included a comparison group matched for age, sex and school, which means that high rates of comorbidity on the autism/obsessive spectrum in the anorexia group cannot be regarded merely as the reflection of the authors' diagnosing autistic-like disorders in great numbers of cases in any population. Third, even though it is always impossible to avoid all bias in diagnosing premorbid problems in a study of this kind (premorbid diagnoses being influenced by previous knowledge of co-existent problems such as anorexia nervosa), the fact that we used blind diagnosis on de-identified case reports with proband and comparison cases mixed, does argue in favour of more valid estimates of premorbid problems in our study than in most earlier research. Finally, we would argue that the possibility of an association between anorexia nervosa and autism spectrum problems has not been revealed previously for the simple reason that it has never been systematically examined before. We set out with the goal of examining all kinds of psychiatric premorbid and comorbid problems in anorexia nervosa and used current definitions of autism, Asperger syndrome and autistic-like conditions. We are aware that some clinicians will say that they have never seen autistic-like problems in anorexia nervosa. We do not contradict that, but would like to emphasize that what you do not look for you will not find.

Apart from the 1985 paper by Gillberg mentioned in the Introduction, there exist at least two further reports on the concurrence of autism and anorexia nervosa. Stiver and Dobbins in 1980 described anorexia nervosa in a girl with autism, highlighting the difficulties encountered in treatment rather than the co-existence of the disorders as such. In the last year, a survey of autism and autistic-like conditions in Tourette syndrome (Comings and Comings, 1990), detailed family pedigrees of Tourette syndrome patients with a relative suffering from autism. In one of these three family case studies, one proband (with Tourette syndrome) had a paternal aunt with anorexia nervosa. The authors did not comment on the co-existence of anorexia in a family with both autism, Tourette syndrome and obsessive-compulsive disorder.

The results of the present study suggest that some underlying trait might be common to Asperger, autisticlike conditions, Tourette, obsessive-compulsive personality disorder and some cases of anorexia nervosa. Superficially, obsessive-compulsive phenomena, are common features of all these conditions. However, these phenomena are not isolated but, in the vast majority of cases referred to here, combined with social and speech/language impairments, usually considered typical of autism and autistic-like conditions. Together, this triad of problems (Wing and Gould, 1979), constitute the bases for making a diagnosis for autism. Could it be that girls with mild autism features (high-functioning autism or Asperger-like) have enough social skills to take them through childhood without major psychiatric disorder or signs of mental breakdown, but not enough to carry them through adolescence into adult life? Could it be that in such girls, anorexia nervosa might be the first severe outward reflection of an underlying autistic-like condition that has been present from early childhood? Could it be that in boys with similar autistic-like problems, the characteristic profile of Asperger syndrome emerges at a much earlier age? Could it even be that the core feature underlying Asperger syndrome does not show such a great male preponderance as previously believed? We found that $1.08 \%$ of all girls under age 18 years suffered or had suffered from anorexia nervosa (Råstam, 1992). Wing found that $1.25 \%$ of all 10 -year-old boys in one English cohort suffered from Asperger syndrome (Wing, personal communication). Diagnosed Asperger syndrome is exceedingly rare in females. Anorexia nervosa is very infrequent in males. Could it be that some female cases of anorexia nervosa represent the mirror image of Asperger syndrome in boys, and that underlying both syndromes in such cases might be a common disorder of empathy? All these questions are speculative and rethorical at the present stage of our knowledge. Nevertheless, the data of the present study indicate the need for asking new questions along these lines in future studies of anorexia nervosa.

Most of the anorexia cases in this study with Asperger syndrome, autistic-like conditions, Tourette syndrome or OCPD showed problems in direct reciprocal social interaction at the time of interview (Råstam, 1992). Many clinicians believe that anorexia nervosa is associated with "alexithymia" or, in other words, an inability to speak about emotional states. We found a similar inability in our cases, but this seemed to us not to be restricted to emotional states, but to mental states generally. Perhaps the alexithymia reported by other authors is just one aspect of 
a deficient theory of mind, which would lead to problems in conceptualizing all mental states, not just those specifically associated with emotions. A deficient theory of mind has been proposed as the basic deficit in autism (Frith, 1989).

The degree to which so many of the anorexia nervosa girls in the present study showed problems reminiscent of high functioning autism and Asperger syndrome was an unexpected by-product of our research. Nevertheless, in retrospect, the findings do seem to make sense. Since our study was not specifically aimed at investigating the contribution of theory of mind problems to the development of anorexia nervosa, conclusions in this domain will have to await further studies focusing specifically on these aspects.

Our final interesting result was the association of dysdiadochokinesis with anorexia nervosa (and particularly with anorexia nervosa with a premorbid history of autisticlike or obsessive-compulsive problems). Recent studies have suggested underlying cerebellar dysfunction in autism (Ritvo et al., 1986; Courchesne et al., 1988). Highly speculatively, dysdiadochokinesis as an isolated neuromotor impairment in teenage girls with anorexia nervosa could be taken to support cerebellar dysfunction in this condition as well.

\section{REFERENCES}

American Psychiatric Association (1987). Diagnostic and Statistical Manual of Mental Disorder, 3rd Edn, revised (DSMIII-R). APA, Washington, DC.

American Psychiatric Association (1980). Diagnostic and Statistical Manual of Mental Disorder, 3rd Edn (DSM-III). APA, Washington, DC.

Baron-Cohen, S. (1989). Do autistic children have obsessions and compulsions? British Journal of Clinical Psychology, 28, 193-200.

Birleson, P. (1981). The validity of depressive disorder in childhood and the development of a self-rating scale: a research report. Journal of Child Psychology and Psychiatry, 22, 73-88.

Comings, D. (1990). Tourette Syndrome and Human Behavior. Hope Press, Duarte, CA, USA.

Comings, D.E. and Comings, B.G. (1992). Clinical and genetic relationships between autism-pervasive developmental disorder and Tourette syndrome: a study of 19 cases. American Journal of Medical Genetics (in press).

Courchesne, E., Yeung-Courchesne, B.A., Press, G.A., Hesselink, J.R. and Jernigan, T.L. (1988). Hypoplasia of cerebellar vermal lobules VI and VII in autism. New England Journal of Medicine, 318, 1349-1354.

Crisp, A. (1980). Let Me Be. Academic Press, London.
Dally, P. (1969). Anorexia Nervosa. Heinemann Medical, London.

Eysenck, H.J. and Eysenck, S.S.G. (1964). Manual of the Eysenck Personality Inventory. University of London Press, London.

Frith, U. (1989). Autism. Explaining the Enigma. Basil Blackwell, Oxford.

Garfinkel, P.E. and Garner, D.M. (1979). The Eating Attitudes Test: an index of the symptoms of anorexia nervosa. Psychological Medicine, 9, 273-279.

Gillberg, C. (1985). Autism and anorexia nervosa: related conditions? Nordisk Psykiatrisk Tidskrift, 39, 307-312.

Gillberg, I.C. (1986). Children with minor neurodevelopmental disorders. Neurological and neurodevelopmental problems at age 10. Developmental Medicine and Child Neurology, 27, 3-16.

Gillberg, C. and Gillberg, I.C. (1989). Asperger syndromesome epidemiological considerations. Journal of Child Psychology and Psychiatry, 30, 631-638.

Littlejohns, C.S., Clarke, D.J. and Corbett, J.A. (1990). Tourettelike disorder in Asperger's syndrome. British Journal of Psychiatry, 156, 430-433.

Olson, D.H., Sprenkle, D.H. and Russell, C.S. (1979). Circumplex model of marital and family systems. Family Process, 18 , 3-28.

Ritvo, E., Freeman, B.J., Scheibel, A.B., Duong, T., Robinson, H., Guthrie, D. and Ritvo, A. (1986). Lower Purkinje cell counts in the cerebella of four autistic subjects: initial findings of the UCLA-NSAC autopsy research report. American Journal of Psychiatry, 143, 862-866.

Rutter, M. (1978). Diagnosis and definition. In Autism. A Reappraisal of Concepts and Treatment (Eds M. Rutter and E. Schopler). Plenum Press, New York.

Rutter, M. (1985). Infantile autism and other pervasive developmental disorders. In Child and Adolescent Psychiatry: Modern Approaches, 2nd Edn (Eds M. Rutter and L. Hersov). Blackwell Scientific, Oxford.

Råstam, M. (1992). Anorexia nervosa in 51 Swedish children and adolescents. Premorbid problems and co-morbidity. Journal of the American Academy of Child and Adolescent Psychiatry, (in press).

Råstam, M. and Gillberg, C. (1991). The family background in anorexia nervosa. A population-based study. Journal of the American Academy of Child and Adolescent Psychiatry, 30, 283-289.

Råstam, M., Gillberg, C. and Garton, M. (1989). Anorexia nervosa in a Swedish urban region: a population-based study. British Journal of Psychiatry, 155, 642-646.

Steffenburg, S. and Gillberg, C. (1986). Autism and autistic-like conditions in Swedish rural and urban areas. A population study. British Journal of Psychiatry, 149, 81-87.

Stiver, R.L.S. and Dobbins, J.P. (1980). Treatment of atypical anorexia nervosa in public school: an autistic girl. Journal of Autism and Developmental Disorders, 10, 67-73.

Wing, L. and Gould, J. (1979). Severe impairments of social interaction and associated abnormalities in children: epidemiology and classification. Journal of Autism and Childhood Schizophrenia, 9, 11-29. 


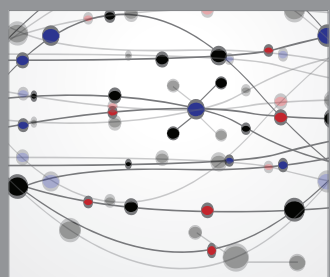

The Scientific World Journal
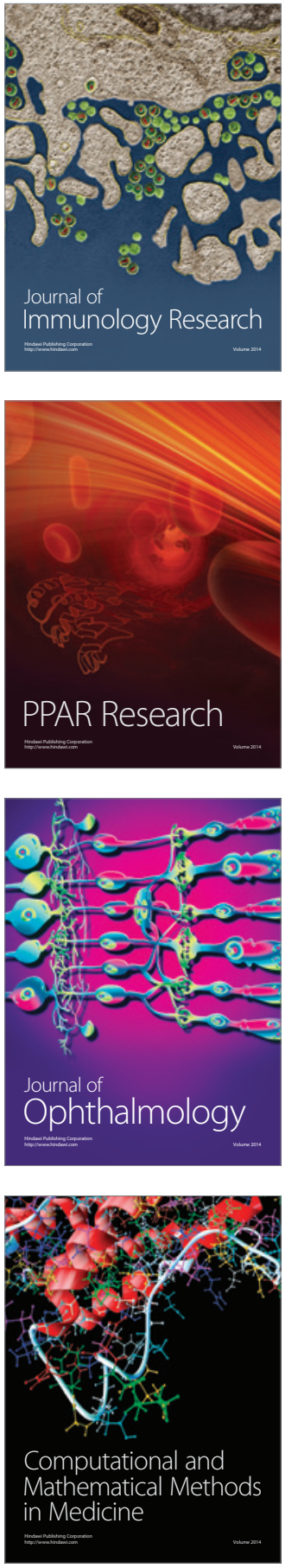

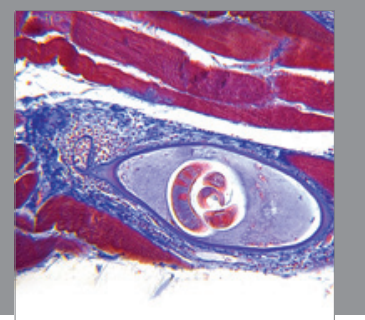

Gastroenterology

Research and Practice
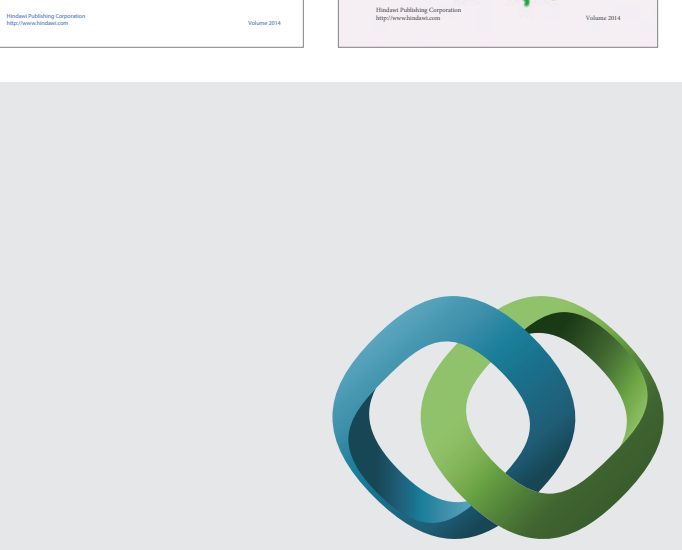

\section{Hindawi}

Submit your manuscripts at

http://www.hindawi.com
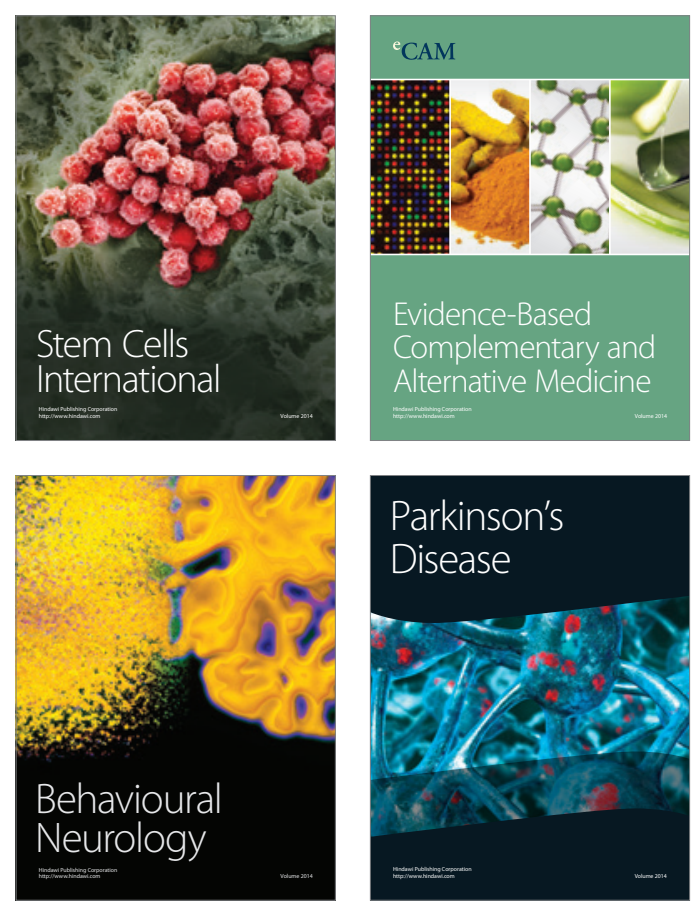

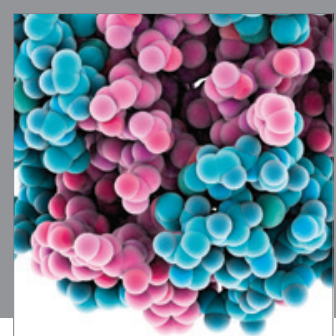

Journal of
Diabetes Research

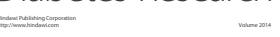

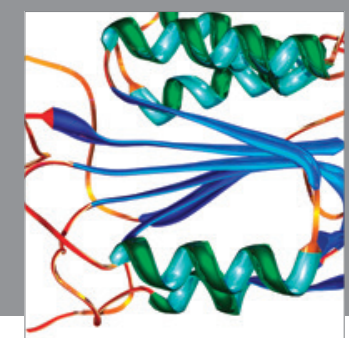

Disease Markers
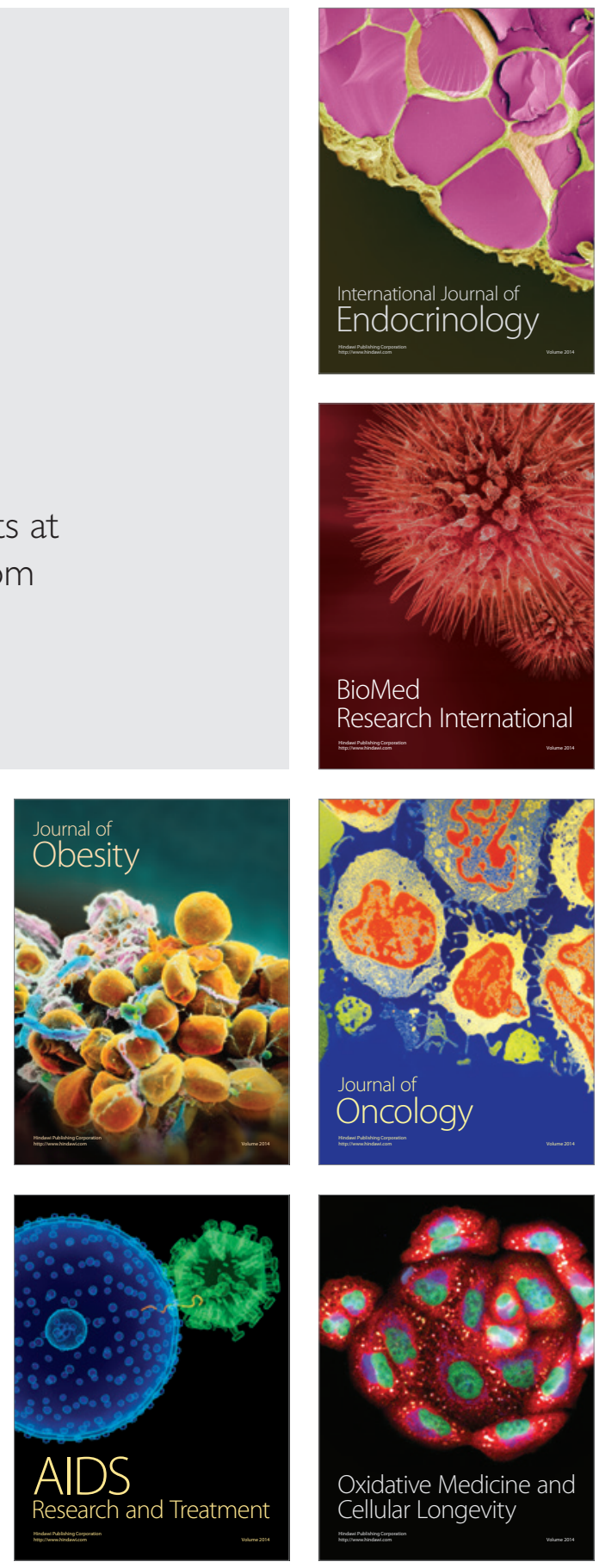